\title{
Desorden del procesamiento auditivo central (DPAC)
}

\author{
Central Auditory Processing Disorder (CAPD)
}

\author{
Oscar Cañete $S^{1}$
}

La audición es un proceso complejo, mucho más de lo que se considera habitualmente. Es así como desde la llegada del sonido al tímpano hasta la percepción de éste, un número importante de operaciones mecánicas y neurobiológicas tienen lugar dentro del sistema auditivo ${ }^{1}$. Desde que la señal choca con la membrana timpánica ésta va siendo sometida a una serie de transformaciones a través de las cuales es convertida en señal eléctrica. Esta señal es transferida desde el oído a través de complejas redes neurales a diferentes áreas del cerebro, para su análisis y posterior reconocimiento o comprensión.

Para la mayoría de las personas cuando alguien se refiere a habilidades auditivas, lo primero que se piensa es en el proceso que ocurre en el oído, es decir, la habilidad de detectar la presencia del sonido $^{2,3}$. Quando se presenta una pérdida auditiva en un individuo se asume que éste ha perdido en forma total o parcial la capacidad de detección de la presencia de sonido, siendo esto realidad. Sin embargo esta capacidad es sólo uno de los muchos procesos que ocurren dentro del sistema auditivo.

Es así como existen individuos que no presentan problemas para detectar la presencia de un sonido, pero sí presentan otras dificultades auditivas como por ejemplo dificultad para comprender conversaciones en ambientes ruidosos, problemas para seguir indicaciones verbales com- plejas, dificultad para aprender nuevo vocabulario o idiomas ${ }^{4,5}$. Estas dificultades pueden afectar tanto el normal desarrollo del lenguaje como el éxito académico o la efectividad comunicativa. $A$ menudo estos individuos no son diagnosticados como pacientes con un problema auditivo debido a que no presentan dificultades en la detección del sonido o no tienen problemas para reconocer el habla en situaciones ideales. Debido a esto se determina que poseen una "audición normal" atribuyéndose estas dificultades a un déficit de atención, problemas de motivación u otra causa.

Después de más de 20 años de estudio de las funciones auditivas, tan solo en el año 1996 la American Speech-Language-Hearing Association (ASHA) estableció un consenso en la definición, procesos de identificación y prácticas de intervención en relación al procesamiento auditivo. Es así como se definió el término Procesamiento Auditivo Central (PAC) como "todos aquellos procesos y mecanismos auditivos responsables de los siguientes fenómenos conductuales: Localización y lateralización del sonido, discriminación auditiva, aspectos temporales de la audición (resolución temporal, enmascaramiento temporal, integración temporal y ordenamiento temporal), Desempeño Auditivo frente a Señales Acústicas Competitivas y Desempeño Auditivo frente a Condiciones de De gradación de la Señal Acústica. Todos estos proce sos y mecanismos son aplicables tanto a señales

\footnotetext{
${ }^{1}$ Tecnólogo Médico, Servicio de Otorrinolaringología, Unidad de Audiología Cínica, Hospital Padre Alberto Hurtado, Santiago.
} 
verbales como no verbales"5,6. La definición anterior es aún objeto de debate entre los expertos, a pesar de esto es la que se considera como la más acertada y completa ${ }^{4,5}$. En palabras simples, el término procesamiento auditivo puede ser definido como "Qué hacemos con lo que escuchamos"6.

\section{NATURALEZA DEL PROCESAMIENTO AUDITIVO CENTRAL (PAC) Y SU ROL EN LA AUDICIÓN}

日 sistema nervioso auditivo central (SNAC) es un sistema complejo con múltiples componentes y niveles, muchos de ellos en paralelo y secuenciales, que a su vez mantienen una organización interactiva. Anatómicamente el SNAC está constituido por núcleos, vías del tronco cerebral, subcorteza, corteza auditiva primaria, corteza de asociación y cuerpo calloso. Mucho de lo que constituye el procesamiento auditivo central es inconsciente, produciéndose lo que se conoce como evento perceptual ${ }^{5,7}$.

A pesar de que el papel del SNAC es crítico en las funciones auditivas que involucran el procesamiento del lenguaje y el procesamiento de otras señales complejas, existen otros factores que participan de igual modo en estas funciones. Por simple que sea una tarea auditiva, ésta se ve influida por funciones de nivel cognitivo superior, como la atención, aprendizaje, motivación, memoria y decisión de procesamiento. La comprensión del lenguaje hablado depende en primera instancia de la detección sensorial inicial así como del análisis perceptual de la señal acústica que es procesada por el SNAC 5 .

Muchos de los aspectos relacionados con la audición (por ejemplo lateralización, localización, tono y tiempo) son mediados por diferentes estructuras y mecanismos de decodificación neural, siendo cada uno de ellos susceptibles a alterarse frente a una patología del Sistema Nervioso Central (SNC). Los parámetros de un estímulo dado son probablemente modificados en forma sucesiva en los diferentes niveles de la vía auditiva central ${ }^{5,8}$.

En forma adicional la plasticidad contribuye a la modificación de las representaciones neurales de un estímulo auditivo. La plasticidad caracteriza a aquellos mecanismos principalmente considerados centrales, siendo la estabilidad una propiedad del sistema auditivo periférico. La plasticidad se refiere a la observación de cambios en la organización del SNAC secundarios a una patología coclear, lesión central, maduración, experiencia, aprendizaje y rehabilitación $3,7-9$.

曰 SNAC es sensible a las diferencias en la estructura del tiempo de una señal acústica (diferencia en el tiempo interaural, ordenamiento temporal, detección del gap). A modo de ejemplo la información temporal es utilizada para la localización, lateralización y ordenamiento temporal así como para la secuenciación ${ }^{2,5}$. 日 SNAC es capaz de detectar y analizar cambios rápidos en el espectro acústico que caracterizan al habla y la música.

Por la individualidad de la organización en la corteza, una misma patología o déficit puede afectar de diferente forma a las funciones auditivas entre individuos de similares características. Eemplo de esto son las diferencias en el desempeño de dos individuos con idéntica pérdida auditiva en el uso de sistemas de amplificación (audífono) ${ }^{10}$.

\section{DESÓRDENES EN EL PROCESAMIENTO AUDITIVO CENTRAL (DPAC)}

La conferencia de Bruton (UTD/Callier Center Concensus Conference) en el año 2000 definió DPAC como un déficit en el procesamiento de la información relacionada en forma específica a la modalidad auditiva, este déficit puede verse exacerbado en ambientes en donde las condiciones acústicas son desfavorables. Estos déficit pueden estar asociados a dificultades auditivas, de comprensión del habla, desarrollo del lenguaje y aprendizaje $e^{11}$.

Un DPAC es resultado de una disfunción en el procesamiento de la información auditiva pudiendo o no coexistir con una disfunción global que afecte el desempeño a través de otras modalidades (déficit de atención, déficit en la representación del lenguaje) ${ }^{1}$. Desórdenes del PAC han sido observados en diversas poblaciones de pacientes, inclui- 
dos aquellos en donde se sospecha de una patología en el sistema nervioso central o un desorden del desarrollo neural (G.: dislexia, trastornos de aprendizaje, trastornos de lenguaje, déficit atencional), es así como también en aquellos en donde un deterioro del sistema nervioso es evidente (E: afasia, esclerosis múltiple, epilepsia, trauma cerebral, tumores, enfermedad de Alzheimer ${ }^{5-7}$. También se ha detectado un DPAC en adultos mayores, presumiblemente debido a cambios neurológicos no patológicos asociados con la edad.

La deprivación auditiva también puede tener efectos perjudiciales sobre la organización y normal desarrollo de las vías auditivas produciendo alteraciones en la maduración de las funciones auditivas centrales. Los efectos de este fenómeno dependen de factores como la edad de instalación y tiempo de duración de tal fenómeno. Un tipo de deprivación bastante frecuente son las otitis me dias recurrentes; se ha podido ver que pérdidas auditivas de tan solo $30 \mathrm{~dB} \mathrm{HL}$ producen efectos sobre el procesamiento auditivo, esto observado en las pruebas conductuales así como en la aparición de latencias prolongadas en Potencial Evocado auditivo de Tronco (PEAT) (en mediciones posresolución de una otitis) en relación a controles $^{12,13}$.

Por lo tanto un DPAC involucraría un déficit en uno o más de los procesos auditivos responsables de las conductas antes señaladas ${ }^{4,5,7}$. Es característico en pacientes con un DPAC la presencia de dificultades de comprensión del lenguaje hablado en ambientes en donde hay ruido de fondo competitivo así como en presencia de reverberación. Niños con un DPAC frecuentemente utilizan las palabras “¿qué?", "¿ah?" en muchas situaciones, demuestran una extrema inatención auditiva te niendo dificultades para poner atención, son fácilmente distráctiles, en muchas oportunidades malinterpretan el mensaje, poseen dificultades para seguir direcciones presentadas como señales auditivas complejas, comandos y presentan problemas en la localización del sonido.

日 diagnóstico de un DPAC se realiza mediante una evaluación audiológica a través de una batería de pruebas, éstas pueden incluir potenciales evocados auditivos así como pruebas conductuales, siendo ambas pruebas administradas bajo condiciones acústicamente controladas. Así también es importante la evaluación mediante patrones de conductas generalmente presentados en forma de encuestas de conductas asociadas ${ }^{2,4}$. Es importante recalcar que el diagnóstico final se realiza una vez descartadas otras posibles causas así como establecido factores que pudiesen influir en esta evaluación. La prevalencia de este desorden varía levemente según diferentes investigadores, los datos más recientes señalan esta variación entre $2 \%$ y $3 \%$ de los niños a una razón de 2:1 entre hombre y mujeres ${ }^{2}$.

\section{MANIFESTACIONES CONDUCTUALES DE UN DPAC}

Existen múltiples conductas que pueden ser observadas en pacientes que poseen un déficit en el procesamiento auditivo. Es importante notar que muchas de estas conductas no son exclusivas de este desorden ya que pueden presentarse en otros déficit o desórdenes como lo son el déficit atencional, pérdida auditiva, problemas conductuales y dificultades de aprendizaje o dislexia ${ }^{14-16}$. Por esto mismo no es recomendable asumir la presencia de un DPAC sólo con la observación de una o más de estas conductas ${ }^{2,3}$ por lo que deben considerarse sólo como conductas de riesgo para ser referido para una evaluación más completa²4,6,7. Ver Tabla 1.

\section{PROBLEMAS ASOCIADOS A UN DPAC}

Desórdenes en el procesamiento auditivo han sido observados en pacientes que manifiestan diversos problemas clínicos. En algunos casos se ha observado en pacientes que padecen de un claro compromiso del sistema nervioso central. Las lesiones cerebrales asociadas con un DPAC pueden situarse en el lóbulo temporal derecho, izquierdo, en el tálamo, los ganglios basales y estructuras del tronco cerebral. Existe un rango de condiciones 
Tabla 1. Conductas observadas en pacientes con un DPAC

- Dificultad para comprender o escuchar en ambientes ruidosos.

- Dificultad para seguir conversaciones largas.

- Dificultad para mantener conversaciones por teléfono.

- Dificultad para aprender un idioma o nuevo vocabulario.

- Dificultad para recordar información hablada (déficit de memoria auditiva)

- Dificultad para tomar notas, dictados.

- Dificultad para mantener la atención en una actividad cuando hay otros ruidos.

- Dificultad para cuando se requiere habilidades organizacionales, ejemplo mantener el orden.

- Dificultad en la lectura y escritura.

- $\quad$ Dificultad en el procesamiento de señales no verbales (ejemplo música)

que incluyen los accidentes vasculares encefálicos, el daño traumático, tumores, epilepsia, enfermedad de Alzheimer, esclerosis múltiple que pueden producir alteraciones en el funcionamiento normal del SNAC2,7.

Una lesión cortical puede afectar el procesamiento auditivo generalmente en forma bilateral. Estudios han demostrado alteraciones en el procesamiento temporal bilateral producto de una lesión o neuropatología en el lóbulo temporal izquierdo y/ o derecho. Lesiones del área temporoparietal dere cha pueden resultar en un déficit bilateral del análisis espectral de una señal. Los déficits contralaterales en la audición dicótica y en la pruebas de discriminación de baja redundancia han sido reportados posteriores a lesiones corticales. Alteraciones en las pruebas electrofisiológicas también han sido observadas en patologías que afectan al SNC o SNAC especialmente en potenciales evocados de latencia media o tardía; pudiéndose encontrarse alteraciones en las amplitudes, morfología y replicabilidad de la respuesta de estos potenciales. Alteraciones tanto en las pruebas conductuales como en las electrofisiológicas han sido observadas cuando existe un compromiso del tronco cerebral y de las vías interhemisféricas ${ }^{5,7}$.

\section{APRENDIZAJE}

La adquisición del lenguaje oral depende del procesamiento de la información acústica. Son precisa- mente los mecanismos centrales los que permiten a los niños aprender el lenguaje oral con rapidez y facilidad. No es de sorprender la estrecha relación entre los DPACy los trastornos en el desarrollo del lenguaje. La mayor evidencia de esta relación se observa en el desempeño de niños con trastornos de aprendizaje que presentan alteraciones en el procesamiento temporal de una señal acústica ${ }^{5}$. Incluso la gramática se hace más vulnerable al déficit de lenguaje en donde las propiedades de la señal necesitan un mayor análisis perceptual. De bemos recordar que existen otros factores que pueden contribuir a un trastorno de aprendizaje por lo que no se puede atribuir el origen de éste sólo a un DPAC. Por ejemplo un déficit en el juego simbólico temprano, pobre memoria inmediata para la ubicación espacial y problemas en la función ejecutiva no tienen conexiones con un DPAC 3,5 . Ver Tabla 2.

\section{USO DEL LENGUAJE}

日 impacto de un DPAC en el lenguaje es particularmente evidente en la comprensión del lenguaje hablado. Adultos con patologías que afectan al SNC y niños con desórdenes en el desarrollo del lenguaje o trastornos de aprendizaje tienen dificultad en la comprensión del lenguaje hablado, incluso cuando poseen un conocimiento de éste. Si alguno de estos individuos además de su condición tuviese un DPAC ciertamente este último 
Tabla 2. Habilidades auditivas importantes en el proceso de aprendizaje

\begin{tabular}{|c|c|}
\hline Discriminación & Diferenciación de sonidos de diferente frecuencia, duración o intensidad. \\
\hline Localización & Ubicación de la fuente sonora. \\
\hline Discriminación auditiva & $\begin{array}{l}\text { Discriminación de los elementos fonémicos del habla que son acústicamente } \\
\text { similares. }\end{array}$ \\
\hline Encierro (Cosure) auditivo & $\begin{array}{l}\text { Comprensión de un mensaje o palabra "completo (a)" cuando una porción de } \\
\text { este (a) está ausente. }\end{array}$ \\
\hline Separación auditiva en ruido & Identificación del hablante primario en presencia de ruido de fondo. \\
\hline Asociación auditiva & Capacidad para otorgar un significado a las palabras. \\
\hline Memoria auditiva & $\begin{array}{l}\text { Capacidad para almacenar y recordar un estímulo en orden o secuencia } \\
\text { apropiada. }\end{array}$ \\
\hline Atención auditiva & $\begin{array}{l}\text { Capacidad para dirigir y mantener la atención hacia una señal acústica } \\
\text { relevante por un período apropiado de tiempo. }\end{array}$ \\
\hline
\end{tabular}

contribuiría de forma importante a sus problemas de comprensión. Es así como pacientes con una lesión en el hemisferio derecho del área temporoparietal presentan dificultad en el análisis de la información acústica así como en la prosodia, la cual ayuda para su comprensión. Es importante considerar que los modelos contemporáneos del uso del lenguaje señalan que la compresión de éste es más que un proceso en donde se analiza la señal acústica. Los oyentes no sólo necesitan identificar o estimar los aspectos de la señal sino también interpretar su valor lingüístico. Se requiere de la activación de la representación léxica, análisis gramatical y juicio del contexto del significado ${ }^{3,5}$.

\section{EVALUACIÓN DEL PROCESAMIENTO AUDITIVO CENTRAL (PAC)}

Una evaluación del PAC tiene como propósito la identificación de la presencia o ausencia de un desorden, la identificación de los procesos afectados y del sitio de la lesión (topodiagnóstico). En conjunto con la información académica y otras mediciones, se desarrolla el perfil de un DPAC. Para lograr ésto se necesita describir el desempeño de las diferentes funciones auditivas ${ }^{5}$.

A pesar que las funciones del procesamiento auditivo parecen ser de gran complejidad, éstas pueden ser evaluadas de diferentes formas. Debido a la individualidad en la organización de la corteza y de las diferentes patologías que pueden afectar tal organización, estas funciones pueden verse afectadas de diferente modo entre individuos. También es muy importante considerar otros factores como edad, nivel de educación, background cultural y social, habilidad cognitiva, uso de medicamentos, etc. En el caso de los niños es importante conocer el estado neuromaduracional del SNAC. Una evaluación del PAC debería proporcionar información tanto de alteraciones en el desarrollo como de desórdenes adquiridos del procesamiento auditivo ${ }^{5-7}$.

日 diagnóstico de un DPAC se logra utilizando diferentes índices, entre los cuales son de importancia:

a) Historia clínica del paciente (enfermedades importantes, problemas de lenguaje, habla, constitución familiar, nivel educacional, cultural, etc),

b) Métodos de observación sistemáticos no estandarizados (Questionarios sobre conductas auditivas, por ejemplo FSHER, CHAPS),

c) Evaluación audiológica (evaluación conductual y/o electrofisiológicay mediciones electroacústicas),

d) Evaluación del habla y lenguaje, y

e) Evaluación médica $2,4,5$. 
Dentro de las pruebas para evaluar el PAC podemos encontrar dos grupos, las pruebas conductuales y las pruebas electrofisiológicas. Las pruebas conductuales corresponden a pruebas audiológicas en donde el estímulo acústico se encuentra manipulado con el fin de estudiar una función auditiva en particular. Dentro de este grupo encontramos por ejemplo pruebas de discriminación de redundancia disminuida en donde las palabras presentadas pueden estar filtradas, comprimidas, etc. También tenemos pruebas de estimulación dicótica presentación simultánea de estímulos diferentes en cada oído), de estimulación biaural (Ejemplo Masking Level Difference Test MLD), procesamiento temporal (por ejemplo Patrones de Frecuencia y Duración) y pruebas de localización y lateralización del sonido (siendo éstas las menos utilizadas clínicamente). Debido al gran número de pruebas existentes es importante contar con una batería que sea lo suficientemente completa sin ser muy extensa ni compleja, en especial al momento de evaluar niños ${ }^{7}$. Se recomienda realizarla a partir de los 6-7 años (pruebas conductuales), debido a que la mayoría de las pruebas están normadas a partir de esta edad; además al ser una evaluación relativamente extensa la mayoría de los niños pequeños pierde interés rápidamente afectando su confiabilidad,2,5-7.

日 otro grupo corresponde a pruebas electrofisiológicas. Estas se refieren principalmente a los Potenciales Evocados de Tronco Cerebral (PEAT) así como los Potenciales Evocados Auditivos de Latencia Media (PLM) y Potenciales Evocados Auditivos de Latencia Tardía (PLT) o también denominados Potenciales Evocados Auditivos Relacionados al Evento2,7. A continuación se describen brevemente algunas de las pruebas más utilizadas en ambos grupos.

\section{PRUEBAS CONDUCTUALES}

Pruebas de Estimulación Dicótica. Involucran la presentación de estímulos auditivos en forma simultánea en ambos oídos, siendo estos estímulos diferentes entre un oído y otro. Se pueden utilizar dígitos, sílabas sin sentido o frases. Dependiendo de la prueba utilizada, el individuo debe repetir todo lo que ha sido escuchado (integración biaural) o dirigir la atención hacia un oído y repetir lo que ha sido escuchado sólo en ese oído (separación biaural). En este tipo de pruebas se ha podido observar lo que se conoce como ventaja del oído derecho, es decir, un mayor rendimiento de este oído, siendo este comportamiento fisiológico debido al cruzamiento de las vías (hemisferio izquierdo dominante para el lenguaje en el $96 \%$ de la población) ${ }^{7}$. En el caso de los dígitos dicóticos se ha visto que son sensibles a lesiones en el tronco cerebral y corteza. La pruebas más utilizadas son: SSW, dígitos dicóticos, disílabos, etc ${ }^{5-7}$.

Pruebas de Redundancia Disminuida. Debido a la presencia de lo que se conoce como redundancia del sistema auditivo (redundancia intrínseca) así como la del lenguaje hablado (redundancia extrínseca), los individuos auditivamente normales son capaces de completar el mensaje y lograr discriminar incluso cuando porciones de la señal auditiva se encuentra ausentes o distorsionadas (encierro auditivo o closure auditivo). Esta función se encuentra afectada en un número importante de pacientes que presentan un DPAC. Modificaciones electroacústicas como alteraciones temporales, frecuenciales o de intensidad producen una disminución principalmente de la redundancia extrínseca. Es muy frecuente que en los individuos que presentan un DPAC se observe un buen rendimiento en la discriminación auditiva en condiciones ideales (ambiente relativamente tranquilo), sin embargo aparecen problemas significativos ante una señal con algún tipo de distorsión. Dentro de las pruebas más utilizadas encontramos discriminación en ruido (por ejemplo identificación de oraciones sintéticas con mensaje de competencia ipsilateral), palabras filtradas (pasa bajo, pasa alto), palabras u oraciones comprimidas (usualmente hasta $60 \%$ de compresión) ${ }^{7}$.

Pruebas de Procesamiento Temporal. Estas pruebas evalúan todos aquellos aspectos relacionados 
con el tiempo de una señal acústica (por ejemplo duración). 日 procesamiento temporal es crítico en una amplia variedad de situaciones de la vida cotidiana, principalmente con la percepción del habla y de la música. Por ejemplo, la percepción de una melodía depende de la habilidad del individuo para percibir el orden de las diferentes notas o acordes así como para determinar si las frecuencias de las notas o acordes son ascendentes o descendentes en relación con las notas adyacentes. En la percepción del habla esta habilidad es necesaria para la discriminación, por ejemplo para la diferenciación de las palabras "pato" y "dato". En la actualidad se utilizan pruebas de patrones de frecuencias, de patrones de duración y de detección del gap (Gap Detection) ${ }^{6,7}$.

Pruebas de Estimulación Biaural. En términos ge nerales evalúan la habilidad del SNAC para procesar por separado, pero en forma complementaria, la información presentada en los dos oídos. A diferencia de las pruebas dicóticas, el estímulo utilizado no es presentado en forma simultánea, pero sí en forma secuencial, o bien la información presentada en cada uno de los oídos es una porción del mensaje completo, de esta forma se necesita de la integración de esta información. Dentro de las pruebas utilizadas tenemos la prueba de fusión biaural y la de diferencia en el nivel de enmascaramiento (Masking Level Difference, $M L D)^{5-7}$.

\section{PRUEBAS ELECTROFISIOLÓGICAS}

日 Mismatch Negativity ( $M M N)$ es un potencial evocado de corteza que surge como resultado de un cambio en la señal acústica dentro de una secuencia repetitiva de sonidos (estímulo oddball). Este reflejaría la discriminación sensorial básica de un estímulo acústico incluso antes de la percepción consciente de la diferencia del estímulo. Este potencial consiste en una onda negativa de 150275 mseg, sus estructuras generadoras serían el tálamo y corteza auditiva ${ }^{7}$. Los individuos que presentan un trastorno de aprendizaje tienden a presentar un pequeño MMN o incluso ausencia de éste tanto frente a un cambio en el estímulo verbal como en uno que no lo es. Niños con trastornos de aprendizaje con alteraciones en el DPAC presentan dificultades en la discriminación de sutiles diferencias fonémicas de un estímulo (ejemplo, /da$\mathrm{ga} /)^{17}$.

Oro potencial de latencia tardía que se ha utilizado clínicamente es el P300, especialmente en pacientes que presentan problemas de aprendizaje. Este se visualiza como una onda positiva cercana a los 300 mseg; para obtener dicha respuesta se utiliza el estímulo oddball. Se han comparado los resultados obtenidos en individuos normales de edad escolar con niños que presentan un DPA. Este último grupo presenta una marcada prolongación de las latencias; las amplitudes eran bastante similares en ambos grupos. Este potencial se ve comprometido en grupos de pacientes que presentan alteraciones en la discriminación auditiva y en niños con problemas de lenguaje receptivo y expresivo. Existen múltiples estudios en donde se ha visto que el P300 sería sensible a los cambios producidos por los tratamientos aplicados a individuos con un DPAC. A pesar de su utilidad es importante recordar que alteraciones en el P300 han sido encontradas en otras poblaciones en donde los resultados anormales no se relacionan con problemas auditivos o comunicacionales, como por ejemplo síndrome de Down, Parkinson o demencia, por lo que para su uso clínico se deben considerar estos factores ${ }^{2}$.

Las pruebas conductuales son las más utilizadas para el diagnóstico de un DPAC debido a que no necesitan de un gran equipamiento, no son complejas de realizar y entregan información sobre la posible ubicación de las lesiones. Es así como por ejemplo una alteración en el lóbulo temporal derecho produce una supresión de las respuestas del oído izquierdo en una prueba dicótica; alteraciones en el cuerpo calloso afectan las pruebas de procesamiento temporal; si se trata del tronco cerebral bajo se evidenciará en las pruebas de integración biaural 7 . Ver Tabla 3.

Cabe señalar que toda evaluación de PAC debe ser precedida por una evaluación audiológica bási- 
Tabla 3. Relación de algunas pruebas de evaluación de Procesamiento Auditivo Central según la estructura de la vía auditiva estudiada

\begin{tabular}{|ccccc|}
\hline $\begin{array}{c}\text { Nervio Auditivo } \\
\text { (Alto) }\end{array}$ & $\begin{array}{c}\text { Tronco Encefálico } \\
\text { (Bajo) }\end{array}$ & Tronco Encefálico & Cortezay Subcorteza & Querpo Calloso \\
\hline PEAT & PEAT & Dígitos Dicóticos & Patrones de frecuencia & Dígitos Dicóticos \\
Reflejo estapedial & MLD & Silabas Dicóticas & Dígitos Dicóticos & Patrones de Frecuencia \\
& Dígitos Dicóticos & Patrones de Frecuencia & SSW & Patrones de Duración \\
& SSW & Fusión Biaural & Habla Flltrada & Oraciones competitivas \\
& Discriminación en ruido & SSW & Habla comprimidacon & \\
& Fusión Biaural & PLM & o sin reverberancia & \\
& Reflejo estapedial & & MLR, P300, MMN & \\
\hline
\end{tabular}

PEAT: Potencial Evocado de Tronco Cerebral. MMN: Mismatch Negativity. PLM: Potencial de Latencia Media MLD: Diferencia en el nivel de enmascaramiento masking level difference). SSW: Staggered Spondaic Word Test

ca, es decir, una audiometría de tonos puros, discriminación auditiva, impedanciometría y en lo posible de emisiones otoacústicas. Esto con el objeto de conocer el estado del sistema auditivo periférico.

Es importante considerar que algunos factores propios del paciente pueden influenciar los resultados de las pruebas de evaluación del PAC; éstos podrían ser: edad, presencia de una pérdida auditiva periférica, síndrome de Déficit Atencional, trastornos del lenguaje o aprendizaje, desórdenes del espectro autista, funciones intelectuales alteradas, entre otros ${ }^{7}$. Las características de las pruebas a utilizar pueden también influir como por ejemplo la sensibilidad, la especificidad, los valores de normalidad, entre otros.

\section{¿QUÉ TIPO DE PACIENTES PUEDE SER EVALUADO?}

Existen varias poblaciones en las que la integridad de las funciones auditivas centrales puede ser estudiada. En un porcentaje alto, los pacientes afásicos pueden presentar un DPAC ${ }^{18}$. La mayoría de las veces un DPAC no es detectado en estos pacientes porque no han sido estudiados desde este punto de vista. Otra patología que puede ser estudiada son las disfunciones neurológicas degenerativas. La enfermedad más común en este tipo de pacientes es la esclerosis múltiple $(\mathrm{EM})^{19}$. Existen evidencias abundantes de un DPAC en pacientes que sufren esta patología cuando las lesiones afectan a las vías auditivas ${ }^{19}$. Se han hallado DPAC en otras enfermedades neurológicas con lesiones en el sistema auditivo como en la enfermedad de Charcot Marie ${ }^{20}$, la enfermedad de Alzheimer ${ }^{21}$, enfermedades degenerativas del sistema olivopontocerebral22, en la ataxia de Federich, en el Parkinson o en algunos tipos de leucodistrofias ${ }^{23}$.

Oro grupo de pacientes en el que la evaluación del PAC puede aportar información significativa son los pacientes hipoacúsicos candidatos a adaptaciones de prótesis auditivas ${ }^{4,10}$. Muchos de estos tienen antecedentes de lesiones en el SNC que podrían afectar su PAC.

La evaluación de las funciones auditivas centrales puede indicar resultados distintos respecto a la simetría observada desde el punto de vista periférico, demostrando la mejor competencia de uno de los oídos. Esto sería particularmente útil para la indicación del oído a rehabilitar en caso de adaptaciones monoaurales $4,5,10$. Por otro lado, en algunos pacientes con amplificación se ha observado un fenómeno denominado de interferencia 
biaural. Este tipo de pacientes se caracterizan por presentar un bajo rendimiento en pruebas de discriminación así como presentar una baja ganancia funcional de la adaptación cuando la amplificación es biaural frente a la monoaural ${ }^{24}$.

Por último en la actualidad otra población en donde se ha podido ver DPAC son aquellos pacientes que presentan trastornos de aprendiza$\mathrm{je}^{3,17,25,26}$. Generalmente estos niños han sido evaluados por múltiples especialistas si haberse clarificado la etiología.

\section{MANEJO}

Estudios recientes en neuroplasticidad sugieren que ésta y la neuromaduración son dependientes (al menos en parte) de la estimulación. De esta forma el manejo de un DPAC debe incluir la estimulación auditiva destinada a producir cambios funcionales dentro del SNAC o SNC. Como ya se señaló, la edad es uno de los factores más importantes a considerar. La pérdida lenta pero continua de neuronas comienza durante la adolescencia continuando a través de los años asociándose a una disminución de la plasticidad. Por ello, los niños se benefician particularmente de una estimulación temprana ${ }^{4,5,7}$. 日 impacto de un DPAC puede variar considerablemente de un individuo a otro, es así como la capacidad de desarrollo e implementación de estrategias compensatorias de termina el nivel de control ante situaciones que representan un reto: las obligaciones educacionales, de trabajo y familia. Para algunos pacientes los síntomas de un DPAC son leves mientras que para otros persisten a pesar del tratamiento oportuno ${ }^{3}$.

Algunos autores describen el manejo de un DPAC como un trípode que se constituye de tres "piernas":

1. Manejo terapéutico directo,

2. Modificaciones ambientales y

3. Estrategias compensatorias.

Las modificaciones ambientales y estrategias compensatorias están diseñadas para mejorar el acceso y uso de la información auditiva por parte del paciente. En contraste las técnicas de manejo están diseñadas para proporcionar una intervención terapéutica directa2,7.

A partir de una evaluación completa del PAC, la rehabilitación de estos desórdenes incluye una intervención dirigida hacia el aumento de la señal acústica, mejoramiento de las capacidades cognitivas y de lenguaje, desarrollo de habilidades, uso de actividades compensatorias y mejoramiento del ambiente auditivo 2,4,5,7,9.

日 propósito de las técnicas terapéuticas es maximizar la neuroplasticidad y mejorar el proce samiento de la información auditiva por parte del cerebro. Tales actividades de tratamiento pueden consistir en técnicas diseñadas para aumentar la discriminación (fonémica), la localización/ lateralización y la prosodia del habla ${ }^{7}$. En los últimos años se ha enfocado la atención a lo que se conoce como entrenamiento auditivo. Reportes recientes han confirmado la utilidad de este entrenamiento como una herramienta de intervención particularmente útil en individuos con trastornos de lenguaje y DPAC. Algunos autores han clasificado el entrenamiento auditivo como formal e informal, el primero realizado por profesionales con un programa determinado y el segundo que se puede realizar en la familia o colegio del paciente ${ }^{9,26,27}$. Existen diferentes técnicas compensatorias que estimulan el desarrollo de las habilidades auditivas; entre las más utilizadas podemos encontrar: discriminación auditiva, análisis auditivo, síntesis fonémica, estrategias para el desarrollo de memoria auditiva, terapia de desensibilización del ruido, estrategias de entrenamiento temporal, entrenamiento dicótico 7,26. Es importante recordar que cada terapia debe estar enfocada a las necesidades individuales de cada paciente, siendo evaluada y analizada en forma periódica

Las modificaciones ambientales están destinadas a mejorar la claridad y aumentar la capacidad de escuchar y oír por parte de un individuo. Se encuentra ampliamente aceptado que el desempeño de un individuo es mejor cuando la señal acústica es clara así como cuando la relación señal ruido $(\mathrm{S} / \mathrm{N})$ es adecuada ${ }^{28}$. 日 manejo de un ambiente puede llevarse a cabo mediante un sistema 
de amplificación $\mathrm{FM}$, otros sistemas de amplificación grupales, modificaciones acústicas de la sala de clases (cambios en los pisos, cielo y ventanas) con el fin de disminuir la distorsión de la señal (por ejemplo el efecto de reverberverancia) ${ }^{3}$.

Fnalmente también es fundamental la labor de los padres y profesores (en el caso de niños) ya que estos puede ayudar a superar las deficiencias producidas por un DPAC mediante, por ejemplo, hablar claro y en forma redundante, ofrecer una ubicación preferencial en la sala de clases, utilizar ayudas visuales como la gesticulación, etc ${ }^{3,7}$.

Es muy importante el trabajo interdisciplinario para el apropiado abordaje y manejo del paciente con un DPAC debido a la diversidad de sus manifestaciones y las consecuencias en el desempeño diario del individuo 29,30 .

\section{CONSIDERACIONES FINALES}

日 estudio del procesamiento auditivo central debe formar parte de la evaluación audiológica de todos aquellos pacientes en donde se sospecha de algún tipo de disfunción o trastorno que pueda tener un origen central. En la práctica médica habitual es frecuente que estos pacientes no sean diagnosticados con un DPAC al no contar con las pruebas apropiadas para la detección de este tipo detrastornos. Muchos pacientes reciben un diagnóstico como hiperactividad, trastorno de lenguaje, síndrome de Déficit Atencional o simplemente se les señala que presentan una "audición normal". 日 acercamiento a este tipo de pacientes puede verse enriquecido con una evaluación, diagnóstico y tratamiento integrados dentro de un equipo multidisciplinario conformado por médicos especialistas, tecnólogos médicos, fonoaudiólogos, terapeutas ocupacionales, psicólogos, educadores, etc.

Si bien el PACy sus desórdenes parecen ser un tema complejo, estudios del sistema auditivo en el área de las neurociencias han proporcionado mucha información útil para la comprensión de estas funciones y de sus trastornos. En otros países, como Estados Unidos, este es un tema ampliamente estudiado por lo que la evaluación y tratamiento de sus desórdenes son conocidos. En nuestro país aún el estudio de estos temas está comenzando, por lo que sería importante sistematizar la evaluación a través del desarrollo de baterías de pruebas, estudiar los efectos en el aprendizaje así como diseñar programas de tratamiento adecuados para nuestra población.

\section{BIBLIOGRAFÍA}

1. Ghermak G Hall III J. Musiek F. Diferencial Diagnosis and Management of CAPD and Attention Déficit Hyperactivity disorder. $J \mathrm{Am}$ Acad Audiol 1999; 10: 289-303.

2. OH田MAK G MUSIEK, F. Central auditory processing disorders: New perspectives. Singular. 1997.

3. Building the link between Hearing, Understanding and Learning. Phonak Hearing Systems, Switzerland.

4. ZENKER F. Barajas JJ. Las funciones Auditivas Centrales (en línea) Auditio: Revista Bectrónica de Audiología. 1 Noviembre2003. vol 2 (2) 31-41 uww.auditio.com/revista/pdf/vol2/2/020203. pdf

5. American Speech-Language Hearing Association (1996). Central auditory processing: Ourrent status of research and implications for clinical practice. American Journal of Audiology, 5 (2): 41-54.

6. KATZ J. Handbook of clinical audiology. $4^{\mathrm{a}}$ edición. Ed. Williams \& Wilkins 1994.

7. BELLIS, T. Assessment and management of Central auditory Processing Disorders in the educational setting. From science to practice. $2^{2}$ edición. Thomas Delmar Learning. 2003.

8. PHШIPS D. Central Auditory System and Central Auditory Processing Disorders. Some Conceptual issues. Seminars in Hearing. 2002; 23 (4): 251-61.

9. Musiek F. SHIN J. HARE C. Plasticity, Auditory Training, and Auditory Processing Disorders. Seminars in Hearing. 2002; 23 (4): 263-75.

10. GVens G ARNOLD T. Auditory processing skills and Hearing aids satisfaction in a sample of older adults. Perceptual and Motor Skills. 1998; 86, 795-801.

11. JgRGR J. Musiek F. Report of the Consensus Conference on the Diagnosis of Auditory Pro- 
cessing Disorders in School-Aged Children. $J$ Am Acad Audiol 2000; 11: 467-74.

12. AsbJornsen A. OBRSUT J. BOLIEK C. MYKING E HOLmeJord A. ReSARTER S Et cols. Impaired Auditory Attentions Skills following middle Ear infections. Child Neuropsychology. 2005; 11: 121-33.

13. HALL JW, GROSE JH. The effects of otitis media with effusion on the masking level difference and the auditory brainstem response. J Speech Hear Res 1993; 36: 210-7.

14. Kraus N, Mogm TJ, Carrell TD et Al. Auditory neurophysiologic responses and discrimination deficits in children with learning problems. Science 1996; 273: 971-3.

15. SCHOCHAT E SOHEVER C. DEANDRADEE ABR and Auditory P300 Fndings in children with ADHD. Arq Neuropsiquiatr 2002; 60 (3-b): 742-7.

16. KING W. LOMBARDINO C. CRANDELL C. LEONARD C. Comorbid Auditory processing disorders in developmental dislexia. Ear \& Hearing . 2003 Oct; 24 (5): 448-56.

17. Banal K. NicO T. ZECKER S. KRAUSS N. Brainstem Timing: Implications for Cortical Processing and Literacy. The Journal of Neuroscience. 2005; 25 (43): 9850-57.

18. DiveMY PL, RoBinson AJ: Nonlinguistic auditory capabilities in aphasia. Brain and Language 1989; 37, 290-326.

19. LeVine R, Gardener J, Stumlmeam S, Fullte ron B, CARLISE E, FurSt N Et AL. Effects of the multiple sclerosis brainstem lesions on sound lateralization and brainstem auditory evoked potentials. 1993; Hearing Research, 68, 73-88.

20. Musiek F. Weder D, Muller R. Audiological finfings in Charcot-Maire-Tooth Disease. Archives of Colaryngology 1982; 108: 595-9.
21. GRIMES AM. GRADY C. FOSTIR NL. SUDELAND T. PATRONAS NJ. Central Auditory Function in Alzheimer's disease. Neurology 1985; 35: 352-8.

22. LYNN GE OLLIS P. GLROY J. OlivopontocerebeIlar degeneration: Effects of auditory brainstem responses. Seminars in Hearing; 4, 375-3

23. KAGA K, TOKORO Y, TANAKA Y, USHIJIMA H. The progress of adrenoleukodystrophy as revealed by auditory brainstem evoked responses and brainstem histology. Arch Atorhinolaryngol 1980; 228: 17-27.

24. WaLDEN T. WALDEN B. Unilateral versus Bilateral Amplification for Adults with Imparied Hearing. J Am Acad Audiol 2005; 16: 574-84.

25. Evaluación del Procesamiento Auditivo Central PAC) y su Aplicación en Trastornos Específicos del Aprendizaje (TEA). Tesista: Echeverry, B. Profesor Guía: Hernán Soto Ramos. Tesis de Magister en Educación Diferencial. Universidad Metropolitana de đencias de la Educación, 1993).

26. BAMION DE, MusieK Æ, LUXON LM. Aetiology and clinical presentations of APD- A review. Ach Dis Child. 2001; 1; 85: 361-5.

27. BARAN, J. Managing auditory disorders in adolescents and adults. Seminars in Hearing, 23 (4), 327-35.

28. Educational Audiology Association. Recommended professional practices for Educational Audiology. Educational Audiology Review, 1997; 13(2): 20-1.

29. PUtigr-Katz H. ADID-Ben SAID L. FEdman I. MIRAND, KUSHNIR DET AL. Treatment and Evaluation indices of Auditory Processing Disorders. Seminars in Hearing. 2002; 23 (4): 357-64.

30. Musiek RM, BARAN JA, SCHOCHAT E Selected management approaches to central auditory processing disorders. Scand Audiol. 1999;28 (suppl 51): 63-76.

Agradecimientos

Dra. Claudia Vinagre D, TO Daniela Fernández O, por su colaboración en la preparación de esta revisión.

Dirección: TM Oscar Cañete S.

E mail: ocanete@hurtadohosp.cl 